\title{
Cirugía micrográfica de Mohs en Uruguay: primeros 130 casos en carcinomas cutáneos
}

\author{
Julio Magliano*, Jorge Navarrete†, Miguel Martínez, Carlos Bazzano§
}

\section{Resumen}

Introducción: la cirugía micrográfica de Mohs es una técnica para la exéresis de cánceres de piel con el examen histológico del $100 \%$ de los márgenes quirúrgicos, logrando la tasa de curación más alta con máxima preservación de tejido sano. Objetivo: realizar una descripción clínico-epidemiológica de nuestras primeras 130 cirugías en el Hospital de Clínicas. Método: análisis descriptivo de todos los pacientes operados por un único cirujano de Mohs en nuestra Unidad de Cirugía Dermatológica desde noviembre de 2013 hasta junio de 2016. Se registraron datos clínicos, tumorales y quirúrgicos.

Resultados: se estudiaron 130 cirugías en 90 pacientes; $62,3 \%$ fueron realizadas en hombres y 37,7\% en mujeres. La edad media fue de 68 años (rango: $33-90$ años). El 67,7\% provenía de Montevideo y el 32,3\% del interior del país. El 68\% correspondía a carcinoma basocelular y $32 \%$ a carcinoma espinocelular. El $91,5 \%$ eran tumores primarios y el $8,5 \%$ recurrentes. El $75,3 \%$ se encontraba en cabeza y cuello. El tipo más frecuente de cierre fueron los colgajos en $43 \%$ (56). Hasta el momento, 70 pacientes han completado seguimiento de al menos un año, donde se vio recidiva tumoral en un solo caso (1/70; 1,43\%). Conclusiones: la cirugía de Mohs es un procedimiento seguro y eficaz, y nuestros resultados coinciden con lo descrito en centros de referencia internacional. Este es el primer centro de Uruguay que cuenta con un cirujano de Mohs y presentamos el primer estudio en pacientes uruguayos.

Palabras clave: CIRUGÍA DE MOHS

NEOPLASIAS CUTÁNEAS

CARCINOMA BASOCELULAR

CARCINOMA DE CÉLULAS ESCAMOSAS

Key words: MOHS SURGERY

SKIN NEOPLASMS

BASAL CELL CARCINOMA

SQUAMOUS CELL CARCINOMA

\footnotetext{
* Profesor Adjunto de la Cátedra de Dermatología.

† Posgrado de la Cátedra de Dermatología.

‡ Profesor Titular de la Cátedra de Dermatología.

$\S$ Profesor Agregado de la Cátedra de Dermatología.

Unidad de Cirugía Dermatológica, Cátedra de Dermatología Médico Quirúrgica.

Hospital de Clínicas Dr. Manuel Quintela. Facultad de Medicina. Universidad de la República. Montevideo, Uruguay.

Correspondencia: Dr. Julio Magliano. Hospital de Clínicas. Av. Italia s/n. Montevideo, Uruguay. Correo electrónico: juliomagliano@gmail.com

Los autores no tienen conflictos de interés.

Fuentes de apoyo financiero: ninguna.

Recibido: 8/11/17

Aceptado: $7 / 1 / 18$
} 


\section{Introducción}

La cirugía micrográfica de Mohs (CMM) es una técnica para la exéresis de cánceres de piel complejos o mal definidos con el examen histológico del 100\% de los márgenes quirúrgicos, logrando la tasa de curación más alta con la máxima preservación de tejido sano circundante $^{(1,2)}$. Combina la excisión quirúrgica y dermatopatología, requiriendo que un único médico actúe en los roles de cirujano y patólogo de manera integrada ${ }^{(2)}$. El hecho que este procedimiento tenga múltiples ventajas por sobre otras terapias convencionales no justifica su uso en todo caso de cáncer de piel, dada la mayor cantidad de tiempo que requiere, los mayores costos y recursos.

Se definen tres zonas de riesgo en el cuerpo: la zona $\mathrm{H}$ de alto riesgo, la zona $\mathrm{M}$ de mediano riesgo y la zona $\mathrm{L}$ de bajo riesgo. La zona $\mathrm{H}$ incluye el área de la "máscara de la cara" (parte central de la cara, párpados [incluyendo canto interno/externo], cejas, nariz, labios [porciones cutánea/mucosa/bermellón], maxilar inferior, oreja y periauricular, genitales, zonas perineal, zona perianal, manos, pies, pezones y areola). La zona M incluye mejillas, frente, cuero cabelludo, cuello y zona pretibial. La zona L comprende tronco y extremidades (excluyendo la zona pretibial, manos y pies) ${ }^{(3)}$. Existen tres medidas de corte, $6 \mathrm{~mm}, 10 \mathrm{~mm}$ y $20 \mathrm{~mm}$. Los tumores de alto riesgo son aquellos que se localizan en la zona $\mathrm{H} \geq 6$ $\mathrm{mm}$, en la zona $\mathrm{M} \geq 10 \mathrm{~mm}$ y en la zona $\mathrm{L} \geq 20 \mathrm{~mm}$. Recientemente, una actualización de las guías de la National Comprehensive Cancer Network (NCCN) considera que un carcinoma localizado en la zona $\mathrm{H}$ ya es de alto riesgo, sin importar el tamaño ${ }^{(4)}$.

Además de su localización y tamaño, determinadas características del paciente o del tumor también pueden llevar a que este sea considerado de alto riesgo, constituyendo indicaciones clásicas de CMM en carcinoma basocelular (CBC) y carcinoma espinocelular $(\mathrm{CEC})^{(3,5)}$ : tumores recurrentes o recidivantes, márgenes clínicos pobremente definidos, pacientes inmunosuprimidos (por ejemplo, trasplantados), aparición en zona de radioterapia previa, invasión perineural demostrada histopatológicamente. Subtipos histopatológicos invasores, para $\mathrm{CBC}$ : morfeiforme, infiltrante, basoescamoso (metatípico), multicéntrico, micronodular, y para CEC: acantolítico, adenoescamoso, desmoplásico o basoescamoso (metatípico).

La CMM se usa principalmente para CBC y CEC; sin embargo, es potencialmente útil en otros tipos de tumores cutáneos ${ }^{(6)}$.

El objetivo general de este estudio es revisar las características epidemiológicas de los 130 primeros casos de carcinomas que tratamos mediante cirugía micrográfica de Mohs en la Cátedra de Dermatología Médico
Quirúrgica del Hospital de Clínicas Dr. Manuel Quintela desde noviembre de 2013 a junio de 2016.

Los objetivos específicos incluyeron el análisis en la población tratada de:

1. Características epidemiológicas.

2. Incidencia de los diferentes tipos de carcinomas cutáneos.

3. Localización de los diferentes carcinomas.

4. Tamaño tumoral.

5. Tipo tumoral según su riesgo.

6. Tamaño del defecto quirúrgico final luego de la CMM.

7. Número de fases requeridas al finalizar la CMM.

8. Origen primario o recurrente.

9. Tipos de reparaciones que se emplearon para la reconstrucción del defecto quirúrgico final.

10. Complicaciones del tratamiento.

11. Seguimiento al año del posoperatorio.

\section{Material y método}

Efectuamos un análisis descriptivo de todos los pacientes que se sometieron a CMM por un único cirujano de Mohs en nuestra Unidad de Cirugía Dermatológica desde el inicio de este procedimiento en noviembre del año 2013 hasta junio de 2016. Puntualmente, para el objetivo específico del seguimiento por una eventual recidiva se expresarán resultados obtenidos hasta el año de seguimiento. Para la obtención de los datos se revisaron las fichas operatorias y las historias clínicas de los pacientes.

Se obtuvieron las siguientes variables: sexo, edad, departamento de procedencia (según residencia), fototipo de Fitzpatrick (I al VI), histopatología, localización según región anatómica y según zona de riesgo (H: alto riesgo, M: riesgo medio, L: riesgo bajo), tamaño de lesión clínica tumoral, número de fases de CMM, tamaño del defecto quirúrgico final, tipo de reconstrucción, origen (tumor primario o recurrente), tratamiento previo recibido (si es que aplica) y complicaciones. Para evaluar las posibles recidivas se tuvieron en cuenta los controles realizados al año de la cirugía.

La CMM se realizó en todos los casos en la Cátedra de Dermatología Médico Quirúrgica del Hospital de Clínicas Dr. Manuel Quintela, en la sala de procedimientos ambulatorios.

Todos los pacientes recibieron previamente la explicación del procedimiento y consintieron por escrito la realización de este, registro fotográfico y su publicación con fines científicos.

Bajo anestesia local se realiza el debulking con bisturí de la lesión y posteriormente se reseca el tumor dejando un margen de 1-2 mm mediante una incisión con un 
ángulo de 45 grados en la periferia y siendo paralela a la superficie cutánea en profundidad.

Se realizan las marcas de referencia sobre la muestra extraída y también sobre el margen quirúrgico. Más tarde se mapea, se cortan y se colorean los márgenes internos de cada fragmento. Cada corte es ordenado, separado y numerado previamente a ser entregado al técnico especialista en Mohs. El técnico coloca cada corte en una platina de congelación, se incluye en gel para congelación, se congelan, se cortan en porciones de 3-5 micras con el criostato y se tiñen con hematoxilina y eosina. El examen de los cortes fue realizado in situ, inspeccionando el $100 \%$ de los márgenes. Si en alguno de los fragmentos persisten células tumorales se realiza una nueva fase, repitiendo el mismo procedimiento exclusivamente en la zona positiva, hasta la extirpación completa del tumor. En la mayoría de los casos la reconstrucción del defecto se realizó bajo anestesia local y en el mismo tiempo quirúrgico.

\section{Resultados}

Se realizaron 130 CMM en 90 pacientes. Durante el año 2013 se realizaron 4 CMM, en el 2014 fueron 10 CMM, en el 2015 se realizaron 70 CMM y en el 2016 se realizaron $46 \mathrm{CMM}$ hasta la fecha mencionada.

El $62,3 \%$ de las CMM fueron realizadas en hombres y $37,7 \%$ en mujeres. La edad media fue de 68 años (rango: 33 - 90 años). El 4,5\% de las intervenciones estuvieron en el rango de 21 a 40 años; en el rango de 41 a 60 años, $21,5 \%$; en el rango de 61 a 80 años, $51 \%$, y en el rango de 81 a 100 años, $23 \%$.

El 67,7\% correspondieron a Montevideo y el 32,3\% al interior del país. Estos últimos se distribuían en los departamentos de Canelones en 57,1\%, Colonia en 11,9\%, San José en $7,1 \%{ }^{(3)}$, Cerro Largo en 7,1\%, Maldonado en $4,8 \%$, Artigas en 4,8\% y Tacuarembó en $2,4 \%$.

En cuanto al fototipo, el $50 \%$ correspondió al tipo III, $43 \%$ al tipo II, $6,2 \%$ al tipo IV y $0,8 \%$ al tipo I. No se registraron fototipos $\mathrm{V}$ y VI.

De las 130 intervenciones realizadas, $68 \%$ correspondieron a CBC y $32 \%$ a CEC. Dentro de los CBC, los subtipos histopatológicos fueron: CBC nodular 89,8\%, CBC superficial 3,4\%, CBC infiltrativo 3,4\%, CBC adenoide $2,3 \%$ y $\mathrm{CBC}$ micronodular $1,1 \%$. Dentro de los CEC, los subtipos histopatológicos fueron; CEC in situ en 14,3\%, CEC bien diferenciado 59,5\%, CEC acantolítico $2,4 \%$, queratoacantoma $19 \%$ y carcinoma cuniculatum $4,8 \%$.

En la zona de alto riesgo $(\mathrm{H})$ se localizaron el $60,8 \%$; $18,5 \%$ en la zona de riesgo medio (M) y el $20,7 \%$ en la zona de riesgo bajo (L). Según la localización anatómica, $73 \%$ se topografiaron en cabeza, $2,3 \%$ en cuello, $15,4 \%$ en extremidades y $9,3 \%$ en tronco. Con respecto a la distribución de los tumores en la cabeza, 22,1\% se localizaron en nariz, $15,8 \%$ en mejilla, $20 \%$ en región periorbitaria, 9,5\% en oreja, $6,3 \%$ en región frontal y $5,3 \%$ se localizaron en la región retroauricular. En cuero cabelludo se topografiaron $4,2 \%$ y el mismo porcentaje se localizó en la sien. En la zona preauricular 3,2\% y en labios 5,3\%. En surco nasogeniano 2,1\% y en mentón y región mandibular $1 \%$ en cada una.

Según la localización anatómica de los CBC, 79,5\% se localizaron en cabeza, $3,4 \%$ en cuello, $9,1 \%$ en extremidades y $8 \%$ en tronco. Con respecto a la distribución de los CBC, específicamente en la cabeza (figura 1), $24,4 \%$ se localizaron en nariz, $21,4 \%$ en región periorbitaria, $13 \%$ en mejilla, $10 \%$ en oreja, $7,1 \%$ en región retroauricular y $5,8 \%$ se localizaron en la región frontal y también en la sien. En labios 4,3\%, en zona preauricular $2,8 \%$ y en surco nasogeniano $2,8 \%$. En cuero cabelludo se localizaron $1,4 \%$ al igual que en mentón con $1,4 \%$.

Según la localización anatómica de los CEC, 59,5\% se localizaron en la cabeza, $0 \%$ en cuello, $28,5 \%$ en extremidades y $12 \%$ en tronco. Con respecto a la distribución de los CEC, específicamente en la cabeza (figura 2), $24 \%$ se localizaron en mejilla, $16 \%$ en nariz, $16 \%$ en región periorbitaria, $12 \%$ en cuero cabelludo, $8 \%$ en oreja, $8 \%$ en región frontal, $8 \%$ en labios, en zona preauricular $4 \%$ y en región mandibular $4 \%$.

El 18,5\% de los tumores tenía un tamaño comprendido entre 0 y $0,5 \mathrm{~cm} ; 21,5 \%$ entre 0,6 y $0,9 \mathrm{~cm} ; 31,5 \%$ entre 1 y $1,9 \mathrm{~cm}, \mathrm{y} 28,5 \%$ entre $2 \mathrm{y}>2 \mathrm{~cm}$.

Con respecto al número de fases, el 68,5\% (89) requirió de una fase; el 22,3\% (29) requirió de dos fases; el $7,7 \%$ (10) requirió de tres fases, y el 1,5\% (2) requirió más de tres fases (figura 3).

Con respecto al tamaño final de los defectos luego de la CMM: el 8,5\% tuvo un tamaño comprendido entre 0 $0,9 \mathrm{~cm} ; 37,7 \%$ entre $1-1,9 \mathrm{~cm} ;$ el $25,4 \%$ entre $2-2,9 \mathrm{~cm}$; el $11 \%$ entre 3 - 3,9 cm; el 9,2\% entre 4 - 4,9 cm; el 2,3\% entre 5 - $5,9 \mathrm{~cm}$; el $2,3 \%$ entre $6-6,9 \mathrm{~cm}$, y el $3,8 \%$ entre 7 - $>7 \mathrm{~cm}$ (tabla 1).

En relación con el tratamiento previo recibido, $91,5 \%$ eran tumores primarios y $8,5 \%$ eran tumores recurrentes. De los tumores recurrentes, siete casos habían sido intervenidos previamente por cirugía convencional, dos casos habían sido intervenidos por CMM (por otro colega), un caso había recibido tratamiento con imiquimod crema y un caso tratado previamente con criocirugía.

En $43 \%$ se usaron colgajos locales (figuras 4 y 5 ); en $35 \%$ se realizó un cierre simple borde a borde; en 16,5\% se realizó cierre por segunda intención; en 3\% se realizaron injertos cutáneos, y en $2,5 \%$ se realizó un cierre combinado. 


\begin{tabular}{|l|c|c|}
\multicolumn{3}{|c}{ Distribución de los CBC en la cabeza } \\
\hline LOCALIZZCIÓN & NUMERO & PORCENTAJES \\
\hline Nariz & 17 & 24,4 \\
\hline Periorbitario & 15 & 21,4 \\
\hline Mejilla & 9 & 13,0 \\
\hline Oreja & 7 & 10,0 \\
\hline Retroauricular & 5 & 7,1 \\
\hline Frontal & 4 & 5,8 \\
\hline Sien & 4 & 5,8 \\
\hline Labio & 3 & 4,3 \\
\hline Preauricular & 2 & 2,8 \\
\hline Surco nasogeniano & 2 & 2,8 \\
\hline Cuero cabelludo & 1 & 1,4 \\
\hline Mentón & 1 & 1,4 \\
\hline
\end{tabular}

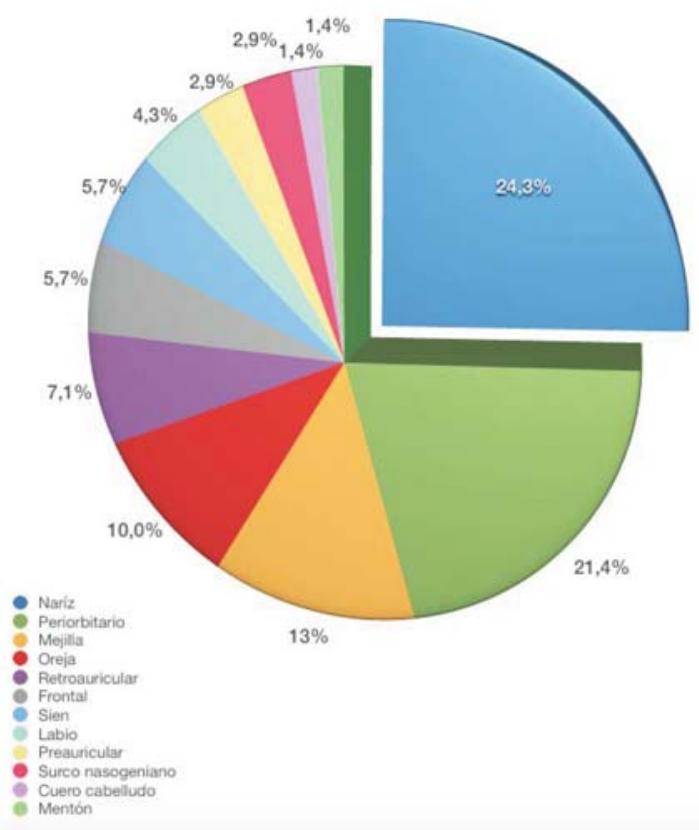

Figura 1. Gráfica donde se representa la distribución de los carcinomas basocelulares en la cabeza.

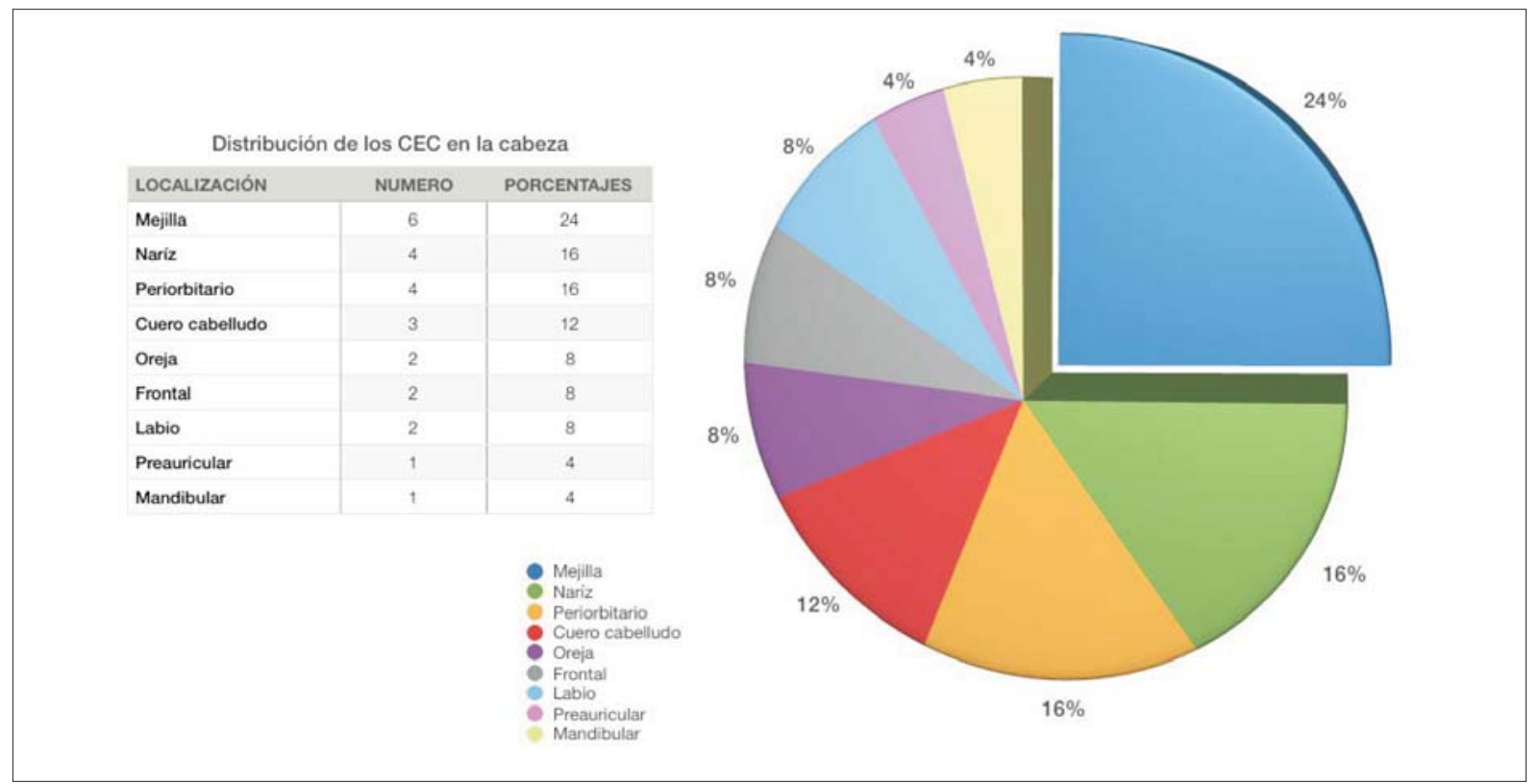

Figura 2. Gráfica donde se representa la distribución de los carcinomas espinocelulares en la cabeza.

Al igual que como se mencionó previamente sobre la localización anatómica de los tumores, los defectos luego de la CMM tuvieron la misma localización: $73 \%$ en cabeza, $2,3 \%$ en cuello, $15,4 \%$ en extremidades y $9,3 \%$ en tronco.
Con respecto a la distribución por áreas, en la cabeza, la nariz fue la zona más reparada con $22 \%$. Aquí se emplearon 13 colgajos, tres cierres simples borde a borde, cuatro por cicatrización por segunda intención y un injerto. 


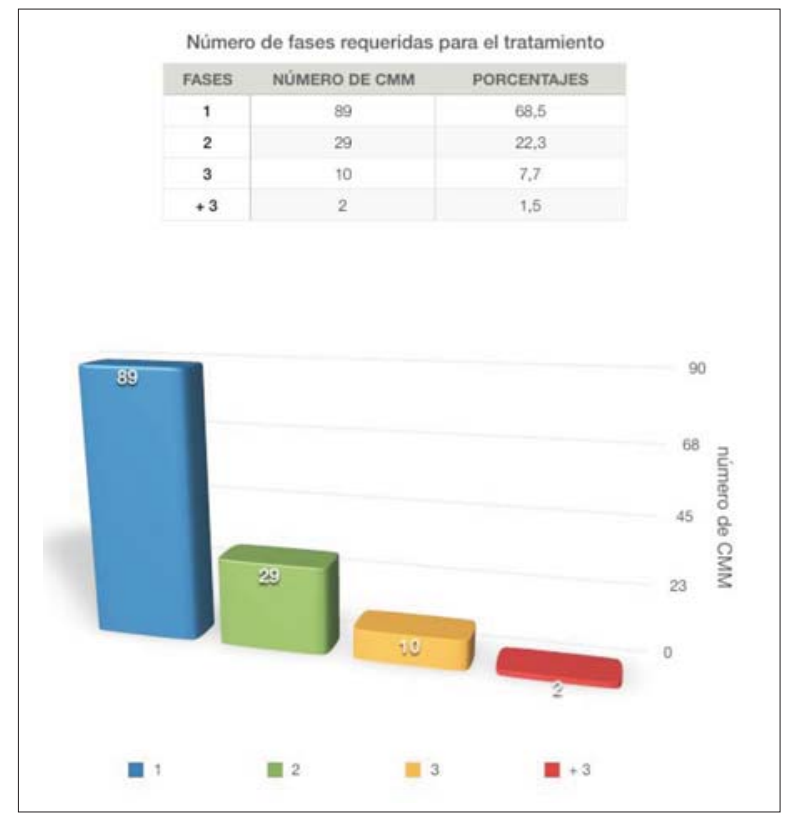

Figura 3. Gráfico donde se representa el número de fases de CMM que se requirió para quedar libre de tumor.

Con respecto a las complicaciones, estas se presentaron en 2,3\% (3/130) de los casos. Las mismas fueron: hematoma con abultamiento de colgajo, necrosis de colgajo y hemorragia posoperatoria. Todas fueron fácilmente corregidas, con remoción de tejido excedente, curaciones y compresión asociado a alginato de calcio, respectivamente. No se presentaron complicaciones infecciosas ni de dehiscencia de las suturas.

Setenta de 130 CMM $(53,85 \%)$ completaron el seguimiento de un año, el tiempo promedio fue de 386 días (rango: 9 días a 40 meses) (tabla 2). Se observó recidiva tumoral en un solo caso de $70(1,43 \%)$, la cual surgió 20 meses posterior a la cirugía. El mismo correspondía a un $\mathrm{CBC}$ nodular primario.

\section{Discusión}

La CMM se considera la técnica más adecuada para todos aquellos tumores considerados de alto riesgo, teniendo en cuenta su localización, tamaño, clínica, histología, recurrencias y estado inmunológico del paciente.

Cuando se trata de comparar las distintas opciones terapéuticas para los carcinomas cutáneos, se encuentran pocos estudios prospectivos, aleatorizados, doble ciego, publicados en la literatura. Sí existen muchos estudios retrospectivos, pero debido a su gran heterogeneidad de variables, como su relación de tamaño, localización y tipo histopatológico de los carcinomas, se hace difícil establecer una conclusión en cuanto a qué opción terapéutica es la más adecuada para determinados casos.
Tabla 1. Tamaño del defecto quirúrgico luego de la CMM.

Tamaño de los defectos quirúrgicos finales

\begin{tabular}{lcc} 
& Frecuencias & Porcentajes \\
\hline $0-0,9 \mathrm{~cm}$ & 11 & $8,5 \%$ \\
$1-1,9 \mathrm{~cm}$ & 49 & $37,7 \%$ \\
$2-2,9 \mathrm{~cm}$ & 33 & $25,4 \%$ \\
$3-3,9 \mathrm{~cm}$ & 14 & $11 \%$ \\
$4-4,9 \mathrm{~cm}$ & 12 & $9,2 \%$ \\
$5-5,9 \mathrm{~cm}$ & 3 & $2,3 \%$ \\
$6-6,9 \mathrm{~cm}$ & 3 & $2,3 \%$ \\
$7->7 \mathrm{~cm}$ & 5 & $3,8 \%$ \\
\hline
\end{tabular}

Tabla 2. Período de seguimiento de los pacientes luego de la CMM.

\begin{tabular}{lcc}
\hline Período de seguimiento & & \\
\hline 0 - 11 meses & Frecuencias & Porcentajes \\
12 - 23 meses & 69 & $53 \%$ \\
24 - 35 meses & 57 & $44 \%$ \\
$>35$ meses & 4 & $3 \%$ \\
\hline
\end{tabular}

Grandes series muestran una predominancia de mujeres entre los pacientes, mientras que en nuestro estudio se vio mayor frecuencia en hombres ${ }^{(7-9)}$. Leibovitch y colaboradores y Ruiz-Salas y colaboradores reportaron un leve predominio de pacientes hombres ${ }^{(10,11)}$.

La edad media de nuestros pacientes fue de 68 años, con un rango comprendido entre 33 y 90 años. Veronese y colaboradores, en su experiencia de 14 años de CMM en CBC de cabeza, reportan una media de edad de 65 años con un rango de 30 a $91^{(12)}$. Macfarlane y colaboradores reportan una media de 69 años con un rango entre 22 y 92 años $^{(13)}$. El mayor porcentaje de las CMM que realizamos fueron en un rango de edad entre los 61 y 80 años, con 51\%. Este dato es concordante con la literatura, ya que los carcinomas, tanto CBC como CEC, se presentan en individuos de mayor edad que otros cánceres de piel como, por ejemplo, el melanoma, que se presenta a edades más tempranas ${ }^{(14)}$.

Con respecto a la procedencia de los pacientes, un gran porcentaje correspondía a pacientes de Montevideo, usua- 


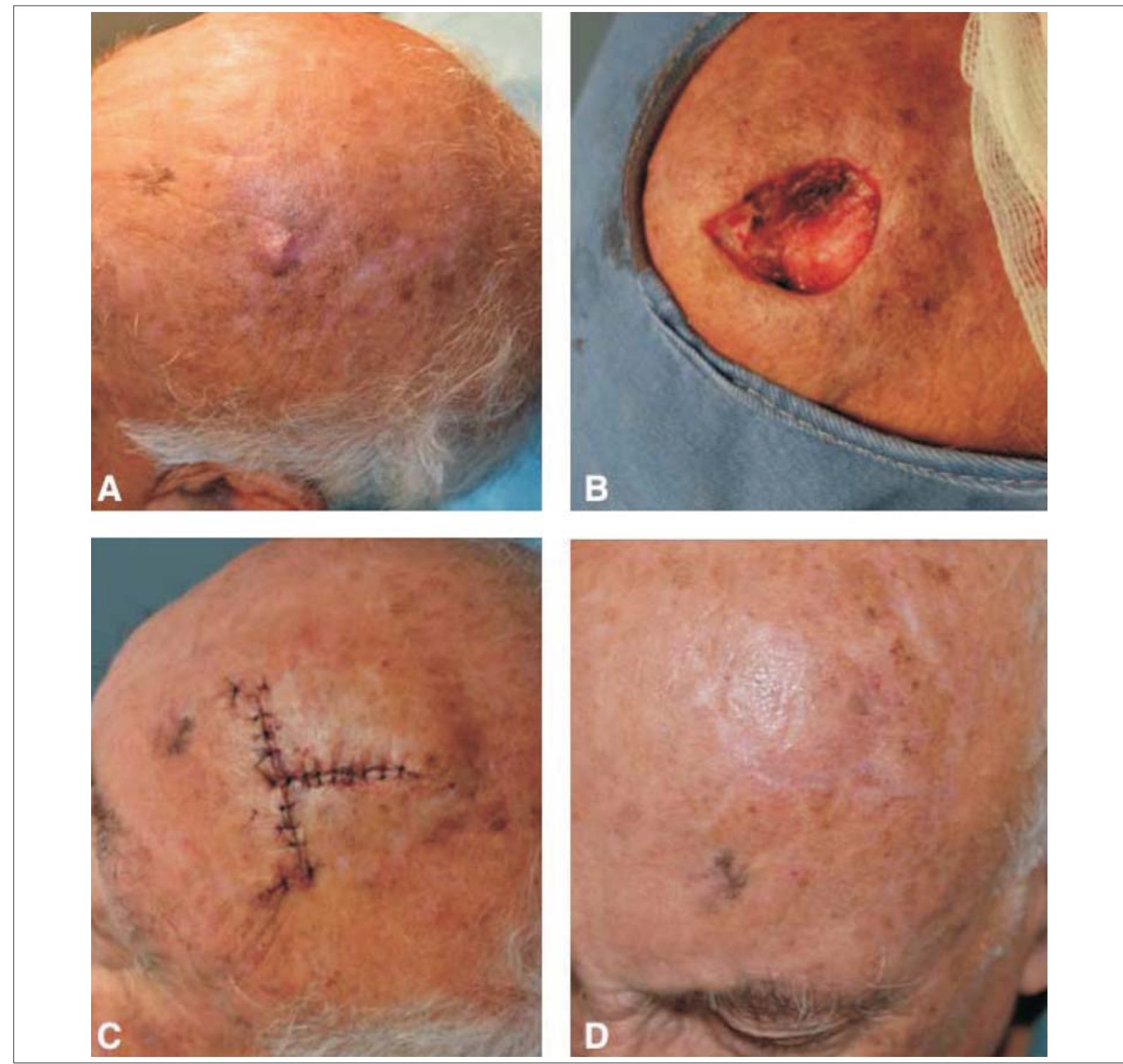

Figura 4. Cierre mediante colgajo OT. A. Carcinoma espinocelular en cuero cabelludo. B. Defecto quirúrgico luego de la CMM. C. Posoperatorio inmediato. D. Tres meses de posoperatorio.

rios del Hospital de Clínicas (67,7\%), pero 32,3\% correspondía a pacientes del interior del país usuarios de salud pública. Esto es importante porque nos habla, en primer lugar, cómo la Cátedra de Dermatología es un centro de referencia en lo que nos concierne a los tumores cutáneos y, por otro lado, la actualización y concientización de los colegas del interior en saber referir a los pacientes que presentan indicación de CMM.

Coincidiendo con la literatura, los carcinomas cutáneos se presentan frecuentemente en los pacientes de piel clara, en nuestros casos el $93 \%$ de los operados con CMM presentaban fototipos de Fitzpatricks bajos (II y III), si bien el más frecuente fue el fototipo III.
Nuestros registros muestran una prevalencia de CBC por sobre CEC; sin embargo, encontramos una cantidad de CEC superior a la esperada (32\%) al comparar con otros estudios similares $(3,54 \%-5,64 \%)^{(11,13-15)}$.

En cuanto a los tipos histopatológicos de los carcinomas, Leibovitch y colaboradores reportan que los subtipos histopatológicos más frecuentes de $\mathrm{CBC}$ fueron el infiltrante con $30,7 \%$, luego el nódulo-quístico con $24,2 \%$ y el superficial con $13,6 \%{ }^{(10)}$. Mientras que para CEC, el bien diferenciado correspondió a 44,4\%, el moderadamente diferenciado a 36\%, el acantolítico a $13,5 \%$ y los pocos diferenciados a $6,4 \%{ }^{(16)}$. Alonso y colaboradores en su trabajo reportan que el subtipo histo- 

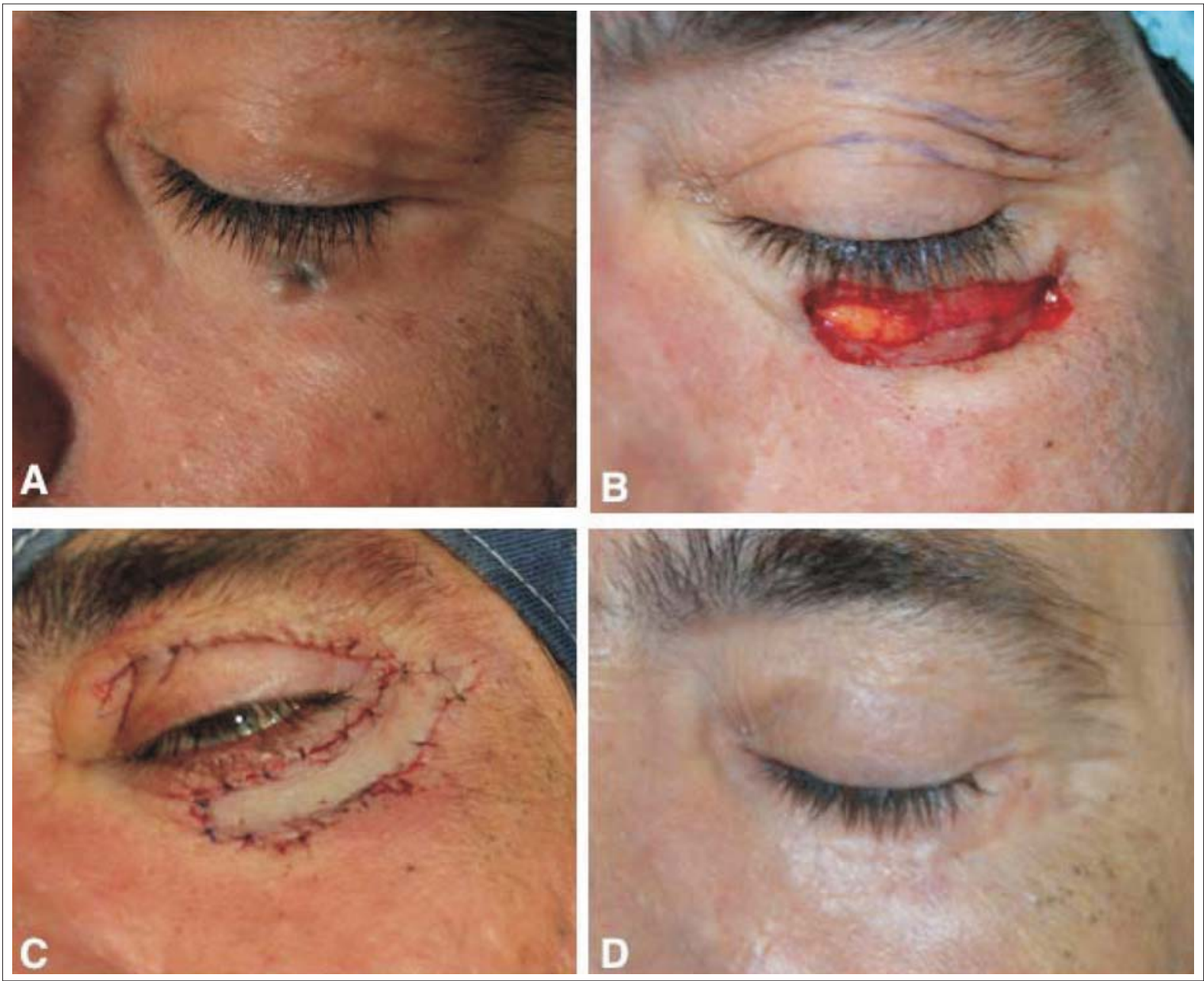

Figura 5. Cierre mediante colgajo de Tripier. A. Carcinoma basocelular en párpado inferior izquierdo. B. Defecto quirúrgico luego de la CMM. C. Posoperatorio inmediato.

D. Seis meses de posoperatorio.

patológico para CBC más frecuente fueron los no agresivos (nodular y superficial) con 52,38\% y los subtipos agresivos (esclerodermiforme y micronodular) con $47,61 \%{ }^{(17)}$.

Con respecto a la localización tumoral en las zonas de riesgo, nuestros resultados también coinciden con la literatura. Macfarlane y colaboradores, en su experiencia de siete años, reportan en $798 \mathrm{CMM}, 39 \%$ de los tumores localizados en el área nasal y $21 \%$ en la región periorbitaria ${ }^{(13)}$. Ibrahim y colaboradores, en su trabajo de reconstrucción de cabeza y cuello, con un número de 245 pacientes, reportan, también coincidiendo con nuestro trabajo, la nariz como la zona que más defectos presentó para la reconstrucción $(53 \%)^{(8)}$. Alonso y colaboradores, en la descripción de sus primeras $100 \mathrm{CMM}$, reportan 52,4\% de CBC localizados a nivel nasal, siendo el sitio más frecuente ${ }^{(17)}$.
El $91,5 \%$ de los carcinomas que intervenimos por medio de la CMM fueron tumores primarios, coincidiendo con Macfarlane y colaboradores, que reportan $72 \%$ de tumores primarios operados ${ }^{(13)}$. También Ruiz Salas y colaboradores reportan cifras aproximadas, donde en su serie de 665 pacientes operados por CMM, el $62 \%$ fueron primarios, $25,04 \%$ fueron recurrencias y $12,37 \%$ fueron persistencia tumoral ${ }^{(11)}$.

Con respecto al tamaño tumoral, en nuestros casos reportamos que para $\mathrm{CBC}$ los tamaños iban de 0,3 a 8 $\mathrm{cm}$, y para CEC de 0,8 a $8 \mathrm{~cm}$. Alonso y colaboradores, en sus primeras CMM, los tamaños de los tumores eran entre 0,7 a $6,5 \mathrm{~cm}$ para $\mathrm{CBC}$ y entre $1,2 \mathrm{~cm}$ y $6 \mathrm{~cm}$ para $\mathrm{CEC}^{(17)}$. Cardoso y colaboradores reportan que el tamaño de las lesiones operadas varió de 0,4 a $12 \mathrm{~cm}$ en el mayor diámetro (promedio de $2,4 \pm 2,0$ ) y 39 pacientes $(50 \%)$ tuvieron lesiones iguales o mayores a $2,0 \mathrm{~cm}$ de diámetro $^{(18)}$. En nuestro trabajo, el 28,5\% de los tumores 
operados estaba en la categoría de mayor o igual a $2 \mathrm{~cm}$, siendo el CEC el más frecuente con 51,3\% y el CBC con $48,7 \%$.

En nuestra serie el número de fases se aproxima a los reportes de la literatura. Macfarlane y colaboradores, en su experiencia de 751 pacientes operados, con un total de 798 CMM, reportan que los tumores que requirieron de una fase para la resolución fueron $58 \%$, de dos fases $34 \%$, de tres fases $6 \%$ y de cuatro fases $1 \%{ }^{(13)}$.

Con respecto al tamaño del defecto quirúrgico en las $130 \mathrm{CMM}$, el $37,7 \%$ presentó un tamaño entre $1 \mathrm{y}$ $1,9 \mathrm{~cm}$ luego de la CMM, pero el $54 \%$ de los defectos fueron mayores de $2 \mathrm{~cm}$. Leibovitch y colaboradores, en su trabajo de 11.127 pacientes con CBC operados con CMM en diez años, señalan que el $41,9 \%$ de los defectos fueron menores a $2 \mathrm{~cm}$ y el $58,1 \%$ fueron mayores a $2 \mathrm{~cm}^{(10)}$. Mientras que en su trabajo para CEC, también de diez años de experiencia, con 1.263 pacientes, los defectos luego de la CMM fueron menores a $2 \mathrm{~cm}$ en $33,9 \%$ y mayores a $2 \mathrm{~cm}$ en $66,1 \%{ }^{(16)}$.

El objetivo de la reparación luego de la CMM es reconstruir el defecto con la cicatriz menos visible. Esto se puede conseguir colocando las cicatrices a lo largo de los márgenes de las unidades cosméticas sobre todo a nivel facial o a lo largo de las líneas de tensión de la piel y reemplazar la piel con igual color, grosor y textura. La reparación de los defectos puede ser un desafío para los cirujanos encargados de la reconstrucción debido al tamaño o localización del defecto ${ }^{(8)}$.

Como mencionamos anteriormente, la mayoría de los defectos se localizaron en la cabeza, y si bien el principal objetivo es el oncológico, es decir curar al paciente, dejando libre de tumor con la CMM, el resultado estético también es importante. Sobre todo debido a que cicatrices inestéticas a nivel facial pueden alterar la calidad de vida de los pacientes.

La frecuencia de métodos reconstructivos descrita para nuestros pacientes se asemeja a lo descrito en estudios más grandes, en donde los colgajos (como grupo) son de preferencia ${ }^{(7,9,19)}$.

Hussain y colaboradores reportan una incidencia de complicaciones menores de $7,78 \%$ en sus CMM, las cuales fueron de características similares a las vistas en nuestros pacientes ${ }^{(15)}$.

El seguimiento al año de la cirugía fue completado en 54\%, con un tiempo promedio de 386 días (rango: 9 días a 40 meses). Se observó recidiva tumoral en un solo caso $(1,43 \%)$, la cual surgió 20 meses posterior a la cirugía y correspondía a un $\mathrm{CBC}$ nodular primario.

Cardoso y colaboradores reportan un período de seguimiento que varió de 6 a 90 meses, y el 75\% de los pacientes fueron seguidos por dos o más años, y $50 \%$ por tres o más años. Solo un paciente $(1,2 \%)$ tuvo recurrencia después de la cirugía de Mohs, que ocurrió luego de 15 meses de seguimiento, y el tipo histológico del tumor fue un $\mathrm{CBC}$ esclerodermiforme ${ }^{(18)}$. Mehrany y colaboradores reportan una incidencia acumulada de recurrencia de $3 \%$ a un año, $12 \%$ a los tres años y $22 \%$ a los cinco años en pacientes con leucemia linfocítica crónica ${ }^{(20)}$. Según el Colegio Americano de Cirugía de Mohs, el período mínimo de seguimiento para todos los tipos de tumores (CPNM de alto riesgo y bajo riesgo) deben ser anuales por cinco años ${ }^{(21)}$. Leibovitch y colaboradores reportan en el seguimiento a cinco años una recidiva de $3,9 \%$ para los CEC $(2,6 \%$ para CEC primarios y $5,9 \%$ para CEC recurrentes) ${ }^{(16)}$. Para este mismo grupo de trabajo, la recurrencia para $\mathrm{CBC}$ fue de $2,6 \%$ a los cinco años $(1,4 \%$ para $\mathrm{CBC}$ primarios y $4 \%$ para $\mathrm{CBC}$ recurrentes $)^{(10)}$. La recidiva a los cinco años fue asociada con un mayor tiempo de duración del tumor antes de la CMM. El sitio más común para la recurrencia fue la nariz $(38,4 \%)$, seguido por la mejilla y el maxilar $(17,1 \%)$ y el área auricular $(10,9 \%)^{(22)}$.

\section{Conclusiones}

Hemos presentado nuestra experiencia en cuatro años en el tratamiento de los carcinomas cutáneos a través de la CMM, brindando de este modo el tratamiento gold standard a pacientes de escasos recursos que concurrieron al Hospital Universitario.

La mayor parte de nuestro perfil clínico-epidemiológico coincide con lo descrito en la literatura.

Hasta el momento solo un paciente ha presentado recurrencia tumoral, sin embargo se requiere de un seguimiento de al menos cinco años para este propósito. Por el momento podemos decir que la técnica de CMM se está realizando de manera segura y con éxito, con resultados comparables a centros internacionales de referencia.

Este es el primer centro de Uruguay que cuenta con un cirujano de Mohs. Una definición del perfil clínico-epidemiológico de nuestros pacientes es necesaria para comparar resultados con otros centros y mejorar constantemente la calidad de atención que se entrega a nuestra población.

\section{Abstract}

Introduction: Mohs micrographic surgery is a technique for the excision of skin cancer with histologic analysis of $100 \%$ of the surgical margins, achieving the highest cure rate, while allowing maximum preservation of surrounding healthy tissue.

Objective: to perform a clinical-epidemiologic description of our first 130 surgeries at the Hospital de Clínicas. 
Method: descriptive analysis of all patients operated by a single Mohs surgeon in our Dermatologic Surgery Unit between November 2013 and June 2016. Clinical, tumoral and surgical data was registered.

Results: 130 surgeries performed in 90 patients were studied. $62.3 \%$ were male patients and $37.7 \%$ female. Mean age was 68 years (range: 33 - 90 years). $67.7 \%$ resided in Montevideo and 32.3\% from other parts of the country. $68 \%$ corresponded to basal cell carcinoma, and $32 \%$ to squamous cell carcinoma. $91.5 \%$ were primary tumors, and $8.5 \%$ were recurrent. $75.3 \%$ were located on the head and neck region. The most frequently used method of closure were flaps in $43 \%$ (56). Up to this moment, 70 patients have undergone follow-up for at least twelve months, and so far, only one case showed recurrence $(1 / 70 ; 1.43 \%)$.

Conclusions: Mohs surgery is safe and effective, and our results agree with reports of international reference centers. This is the first center in Uruguay with a Mohs Surgeon, and we present the first study in Uruguayan patients.

\section{Resumo}

Introdução: a cirurgia micrográfica de Mohs é uma técnica para a exéreses de cânceres de pele com o exame histológico de $100 \%$ das margens cirúrgicas, conseguindo a taxa de cura mais alta com máxima preservação de tecido saudável.

Objetivo: realizar uma descrição clínico-epidemiológica das primeiras 130 cirurgias realizadas no Hospital de Clínicas de Uruguai.

Método: análise descritiva de todos os pacientes operados por um único cirurgião de Mohs na Unidade de Cirurgia Dermatológica no período novembro 2013 junho 2016. Foram registrados dados clínicos, tumorais e cirúrgicos.

Resultados: foram analisadas 130 cirurgias realizadas em 90 pacientes sendo. $62.3 \%$ em homens e $37.7 \%$ em mulheres. A média de idade média foi 68 anos (intervalo: 33 - 90 anos). $67.7 \%$ eram originários de Montevidéu e os restantes $32.3 \%$ do interior. $68 \%$ correspondiam a carcinoma basocelular e $32 \%$ a carcinoma de células escamosas. $91.5 \%$ eram tumores primários e $8.5 \%$ recorrentes. $75.3 \%$ estavam localizados em cabeça e pescoço. O tipo mais frequente de fechamento foi por retalhos em 43\% (56). Até o momento, 70 pacientes completaram um seguimento mínimo de 1 ano, no qual se observou recidiva tumoral em somente 1 caso (1/70; $1.43 \%)$.

Conclusões: a cirurgia de Mohs é um procedimento seguro e eficaz, e nossos resultados coincidem com os descritos por centros de referência internacional. Este é o primeiro centro no Uruguai que conta com um cirurgi- ão de Mohs e que apresenta o primeiro estudo em pacientes uruguaios.

\section{Bibliografía}

1. Benedetto PX, Poblete-López C. Mohs micrographic surgery technique. Dermatol Clin 2011; 29(2):141-51.

2. Connolly SM, Baker DR, Coldiron BM, Fazio MJ, Storrs PA, Vidimos AT, et al. AAD/ACMS/ASDSA/ASMS 2012 appropriate use criteria for Mohs micrographic surgery: a report of the American Academy of Dermatology, American College of Mohs Surgery, American Society for Dermatologic Surgery Association, and the American Society for Mohs Surgery. J Am Acad Dermatol 2012; 67(4):531-50.

3. Blechman AB, Patterson JW, Russell MA. Application of Mohs micrographic surgery appropriate-use criteria to skin cancers at a university health system. J Am Acad Dermatol 2014; 71(1):29-35.

4. Bichakjian CK, Olencki T, Aasi SZ, Alam M, Andersen JS, Berg D, et al. Basal Cell Skin Cancer, Version 1.2016, NCCN Clinical Practice Guidelines in Oncology. J Natl Compr Canc Netw 2016; 14(5):574-97.

5. Goldenberg A, Ortíz A, Kim SS, Jiang SB. Squamous cell carcinoma with aggressive subclinical extension: 5-year retrospective rewiev of diagnostic predictors. J Am Acad Dermatol 2015; 73(1):120-6.

6. Ocampo-Candiani J, Vidaurri LM, Olazarán Medrano Z. Cirugía micrográfica de Mohs en tumores malignos de la piel. Med Cutan Iber Lat Am 2004; 32(2):65-70.

7. Grosfeld EC, Smit JM, Krekels GA, van Rappard JH, Hoogbergen MM. Facial reconstruction following Mohs micrographic surgery: a report of 622 cases. J Cutan Med Surg 2014; 18(4):265-70.

8. Ibrahim AM, Rabie AN, Borud L, Tobias AM, Lee BT, Lin SJ. Common patterns of reconstruction for Mohs defects in the head and neck. J Craniofac Surg 2014; 25(1):87-92.

9. Wain RA, Tehrani H. Reconstructive outcomes of Mohs surgery compared with conventional excision: A 13-month prospective study. J Plast Reconstr Aesthet Surg 2015; 68(7):946-52.

10. Leibovitch I, Huilgol SC, Selva D, Richards S, Paver R. Basal cell carcinoma treated with Mohs surgery in Australia I. Experience over 10 years. J Am Acad Dermatol 2005; 53(3):445-51.

11. Ruiz-Salas V, Garcés JR, Miñano Medrano R, Alonso-Alonso T, Rodríguez-Prieto MÁ, López-Estebaranz JL, et al. Descripción de los pacientes intervenidos mediante cirugía de Mohs en España: datos basales del registro español de cirugía de Mohs (REGESMOHS). Actas Dermosifiliogr 2015; 106(7):562-8.

12. Veronese F, Farinelli P, Zavattaro E, Zuccoli R, Bonvini D, Leigheb G, et al. Basal cell carcinoma of the head region: therapeutical results of 350 lesions treated with Mohs micrographic surgery. J Eur Acad Dermatol Venereol 2012; 26(7):838-43. 
13. Macfarlane L, Waters A, Evans A, Affleck A, Fleming C. Seven years' experience of Mohs micrographic surgery in a UK centre, and development of a UK minimum dataset and audit standards. Clin Exp Dermatol 2013; 38(3):262-9.

14. Lee DA, Miller SJ. Nonmelanoma skin cancer. Facial Plast Surg Clin North Am 2009; 17(3):309-24.

15. Hussain W, Affleck A, Al-Niaimi F, Cooper A, Craythorne E, Fleming C, et al. Safety, complications and patients' acceptance of Mohs micrographic surgery under local anaesthesia: results from the UK MAPS (Mohs Acceptance and Patient Safety) Collaboration Group. Br J Dermatol 2016; 176(3):806-8.

16. Leibovitch I, Huilgol SC, Selva D, Hill D, Richards S, Paver R. Cutaneous squamous cell carcinoma treated with Mohs micrographic surgery in Australia I: experience over 10 years. J Am Acad Dermatol 2005; 53(2):253-60.

17. Alonso T SnP, González A, Ingelmo J, Ruiz I, Delgado S, Rodríguez M. Mohs micrographic surgery our first 100 patients. Actas Dermosifiliogr 2008; 99(4):275-80.

18. Cardoso F DSB. Mohs micrographic surgery: a study of 83 cases. An Bras Dermatol 2012; 87(2):228-34.
19. Hafiji J, Hussain W, Salmon P. Mohs surgery spares the orbicularis oris muscle, optimizing cosmetic and functional outcomes for tumours in the perioral region: a series of 407 cases and reconstructions by dermatological surgeons. Br J Dermatol 2015; 172(1):294-6.

20. Mehrany K, Weenig RH, Pittelkow MR, Roenigk RK, CC. O. High recurrence rates of Basal cell carcinoma after mohs surgery in patients with chronic lymphocytic leukemia. Arch Dermatol 2004; 140(8):985-8.

21. Leitenberger JJ, Rogers H, Chapman JC, Maher IA, Fox MC, Harmon CB, et al. Defining recurrence of nonmelanoma skin cancer after Mohs micrographic surgery: Report of the American College of Mohs Surgery Registry and Outcomes Committee. J Am Acad Dermatol 2016; 75(5):1022-31.

22. Leibovitch I, Huilgol SC, Selva D, Richards S, Paver R. Basal cell carcinoma treated with Mohs surgery in Australia II. Outcome at 5-year follow-up. J Am Acad Dermatol 2005; 53(3):452-7. 